\title{
Case-finding Effectiveness of HIV Partner Notification Service in a High-volume Facility in North-Central Nigeria
}

\author{
Adebayo Opeyemi ${ }^{1,2 *}$, Ugwuanyi Charles ${ }^{3}$, Adegoke Adetola ${ }^{1}$ \\ ${ }^{1}$ Department of Prevention Care and Treatment, Institute of Human Virology, Nigeria \\ ${ }^{2}$ Special Treatment Clinic, University of Abuja Teaching Hospital, Nigeria \\ ${ }^{3}$ TB Unit, Johns Hopkins University Centre for Communication Programs, Nigeria
}

\begin{abstract}
Partner Notification Service (PNS) has been a long-standing component of the HIV/AIDS control programs and a keep part of the innovative approaches to achieve epidemic control. Many programs around the world and facilities, especially in Nigeria, have been scaling up the strategy. The study reviewed the activities of Partner Notification Services in the University of Abuja teaching hospital, Abuja, between January 2019 and December 2019. A retrospective cohort analysis of client's folders, registers, and the electronic medical record was done. A total of 451 clients with HIV infection (194 New Positives and 257 Virally unsuppressed clients) were interviewed. The majority of the clients (86.2\%) accepted the offer of Partner Notification Service and elicited 457 partners (1: 1.2 partners), 388 (84.9\%) were notified of their risk of exposure to HIV. Of the 323 partners that were reached, 97 (30\%) previously knew their HIV-positive status, 62 (19.2\%) were newly diagnosed, and 164 (50.8\%) tested non-reactive. The total HIV testing yield was 3.8\% (207 positives from 5394 HIV test done), Index testing done through Partner Notification Service had the highest yield at 27.4\%, then Voluntary testing and counselling at 10.1\%, followed TB clinic HTS at a yield of 2.9\%. Other testing points had a yield of $\leq 1 \%$. The study supports many other similar studies in demonstrating the effectiveness of Partner Notification service as a key strategy in achieving epidemic control of HIV/AIDS. It is also effective in the management of scarce resources that currently plagues many developing nations like Nigeria.
\end{abstract}

Keywords: HIV Testing Services, Index Testing, Partner Notification Services.

\section{Introduction}

The first case of HIV/AIDS in Nigeria was reported in 1986. Since then, the national prevalence has increased from $1.8 \%$ in 1991 , peaking at $5.8 \%$ in 2001 , and progressively reducing to $3.1 \%$ in $2014[1,2]$. In 2019 , the Nigeria National HIV/AIDS Indicator and Impact Survey (NAIIS) reported a national prevalence of $1.4 \%$. The South-South zone of the country has the highest prevalence $(3.1 \%)$, then $2.0 \%$ in the North Central zone, and $1.9 \%$ in the South-East zone. HIV prevalence is lower in the South-West zone (1.1\%), $1.1 \%$ in the
North-East zone, and $0.6 \%$ in the North-West [3, $4,5]$.

HIV testing services (HTS) are very crucial to achieving the first 90 of the United Nations 9090-90 targets to end the HIV epidemic, meaning $90 \%$ of people living with HIV should learn their HIV status. About $25 \%$ of people with HIV are unaware of their infection, and the only way to determine a person's HIV status is for them to have an HIV test [6]. To bridge this testing gap, there has been the rapid expansion of HIV testing and treatment services, with different innovative approaches being developed and introduced to expand the HIV testing services in 
other to reach the "fast-track" goals by 2020 [7].

HIV testing services include the full range of services provided alongside HIV testing: Pre and Post-test counselling, linkage to care, and treatment. HIV Counselling and Testing (HCT) remains the entry point for prevention treatment, care, and other support services for person's living with HIV/AIDS. In the light of dwindling resources, multiple approaches, including clientinitiated and provider-initiated counselling and testing, have been utilized for targeted demand creation $[8,9]$.

Targeted HIV testing refers to innovative strategies that increase yield in HIV testing, especially among key subpopulations that are hard to reach. Specific population groups are appropriate for targeted testing because they are either at high risk of HIV infection or risk of onward transmission if they are HIV positive or less likely to access routinely offered HTS. These groups include:

- Key populations: Sex workers (SW), Men who have sex with men (MSM), Transgender people (TG), People who inject drugs (PWID), People in prisons or other closed settings.

- Vulnerable populations: Adolescent girls and young women (AGYW), Adolescent boys, Men especially young men, Migrant and itinerant workers.

- Sexual and drug-injecting partners of PLHIV.

- HIV-exposed infants.

Distinct approaches are ideal for the distinct populations mentioned earlier, depending on the setting, health facility, and community-based collaborators. However, in this study, our attention is on the subset of sexual (and or druginjecting) partners of PLHIV, which refers to Partner Notification Services. The sexual and drug-injecting partner's persons diagnosed and living with HIV infection have an increased probability of being HIV-positive. Partner Notification Services refers directly to the testing of Sexual and drug-injecting partners of PLHIV, a simple and yet effective way of reaching many of these partners who are undiagnosed and sometimes unaware of their HIV exposure [10, 11].

Partner notification service has been an important public health approach for the management of various infectious diseases for decades, including programmes for sexually transmitted infections and tuberculosis. However, its implementation for PLHIV is not routine [7]. All partner notification testing should ensure they meet the WHO's 5 Cs (Consent, Confidentiality, Counselling, Correct test results, and Connection to Care and Treatment Services) minimum criteria. It should be voluntary and non-coercive. Partner notification is also not a one-time event but rather offered routinely at HIV diagnosis, at least annually as part of HIV treatment services and after any change in relationship status [12]. A conscious effort needs to be made to minimize intimate partner violence (IPV) risk following partner notification. i.e., partner notification should take a "do no harm" approach. Therefore, the initial screening of the index partner should include an assessment of the risk for IPV. Thus, partner notification may be contraindicated in those who report IPV or fear IPV or other forms of social harm following the disclosure of HIV status [13].

Different approaches may be considered in the practice of partner notification, including the passive and the assisted HIV partner notification. In the passive HIV partner notification, a trained provider encourages the index partner to disclose their HIV status to their sexual or drug-injecting partners and suggests the need for HIV testing service to such partners. The index partner is thus responsible for notifying the partner and referring them for HTS. This is the "standard of care" recommended in 2015 WHO HTS guidelines [14].

However, the assisted HIV partner notification services are such that the provider helps the consenting index partner notify their sexual or drug-injecting partners of the index partner's HIV status and recommends HTS. 
Such notification may occur with the disclosure of the index client's identity and HIV status or anonymously, without the disclosure of the index's identity. Assisted partner notification [15] uses the provider referral (with the consent of the index partner, a trained provider confidentially contacts the person's partner(s) directly and offers voluntary testing), Dual referral (the trained health provider accompanies and provides support to the index patient while they disclose their HIV status, and offers voluntary testing to the partner(s), and the Contract referral (the index patient agrees to enter into a contract with a provider to disclose their status within a specified timeframe. If the partner(s) of the HIV-positive individual don't access testing or contact the health provider within that period, then the provider will contact the partner(s) directly and offer them voluntary testing). Couple HTS encourages couples to come for testing together and to disclose their HIV status to each other during the counselling session. The approach leads to improved outcomes such as linking couples to care, increasing adherence to treatment and uptake of PMTCT service, and the reduction of IPV and stigma $[13,16]$.

The primary objective of the study is to evaluate the case-finding effectiveness of HIV partner notification services in the University of Abuja Teaching Hospital (UATH), Gwagwalada, Abuja, Nigeria, and compare its positivity yield (Number of the test to be done to get a positive client) with other testing streams in the facility. The information will inform the redistribution of scarce resources, including but not limited to manpower, test kits, and other consumables to testing streams with more yield.

\section{Materials and Methods}

This is a retrospective cohort study that analysed and reviewed data collected from index cases and their sexual partners who received PNS between January 2019 and December 2019 in the University of Abuja Teaching Hospital, Abuja, Nigeria. It also compared the testing data from Index to other testing streams - Voluntary Counselling and Testing (VCT), Provider Initiated Counselling and Testing (PITC), testing at Antenatal Clinic (ANC), Inpatient Testing, Tuberculosis (TB) unit, and Emergency unit. The study was carried out to inform processes and evaluate the outcomes of processes in the facility.

\section{Study Background and Setting}

University of Abuja Teaching Hospital (UATH) is located 45 minutes from downtown Abuja, in the Gwagwalada Area Council of the Federal Capital Territory (FCT). Its foundation was laid in 1981 and was commissioned in 1992, having 360-beds and a plan to expand to 500 beds. It was originally established as a General Hospital and later upgraded to a Specialist Hospital (1993), then a Teaching Hospital in 2006. The hospital serves as a reference Hospital for FCT and the surrounding states.

The University of Abuja Teaching Hospital was the first site to partner with the Institute of Human Virology Nigeria's AIDS Care and Treatment in Nigeria (ACTION) program (now ACHIEVE program) to receive PEPFAR support. It has grown into one of the largest providers of HIV care, treatment, and support services since February 2005.

The ART clinic is staffed by a full range of consultants, medical officers, dedicated nurses, adherence counsellors, pharmacists, and PLHIV's employed as treatment support specialists. The 500-bed hospital also houses the IHVN's Clinical Training Centre built with the support of both the FMOH and CDC to train physicians, nurses, and ancillary health care workers to provide high-quality and effective comprehensive, multidisciplinary care and treatment services for PLHIVs and meet the growing need for health-worker training in Nigeria.

The Centre houses a multidisciplinary (adult and paediatric) care clinic with 12 dedicated consulting and patient examination rooms, nursing and counselling cubicles, pharmacy 
dispensary, and training room with conference facilities. The University of Abuja Teaching Hospital had 5350 clients on treatment as of $31^{\text {st }}$ December 2019. The number of adults on treatment is 4,915 , and pregnant women on treatment (a subset of adults) are 8. It offers the following services with support from the Institute of Human Virology Nigeria (IHVN).

- HIV Counselling and Testing including Partner Notification Services.

- Prevention of Mother to Child Transmission

- Prevention with Positives.

- Adult ARV treatment services.

- Paediatric ARV treatment services.

- Opportunistic Diseases diagnosis and management.

- TB/HIV collaborative services.

- Laboratory Services.

- Basic Care and Support (Adults and Children including OVC).

- Home Based and Community Care.

- Promotion of safe blood and injection practices.

Gracious Support Group for PLHIVs meet in the community, adolescent support group, and a mother-to-mother support group thrive at UATH. Over 80 clients are home-based care volunteers who volunteer on home-based care teams to provide adherence, palliative, and basic care service to patients in the community. Service delivery is supported by extensive training programs, health systems information systems for monitoring and evaluation, and sitecentered quality assurance and improvement programs. Scale-up and integration of services have been conducted through collaboration and capacity building with the National Agency for the Control of AIDS (NACA) and the Federal Ministry of Health.

\section{Study Population}

The study population included persons (15 years and above) who were tested positive for HIV infection and accepted PNS between January 2019 to December 2019. They included positives identified in the Voluntary and Counselling unit (VCT), Antenatal clinic (ANC), TB clinic, Provider Initiated Counselling and Testing (PITC), Emergency unit, and Inpatient wards of the University of Abuja Teaching Hospital. They also included clients with unsuppressed viral load levels (clients on HIV treatment services but with viral load levels greater than $1000 \mathrm{cp} / \mathrm{ml}$ after at least 6 months on ARVs) within the same review period.

\section{Partner Notification Procedures and Data Collection}

Medical records (paper-based and EMR with keen attention to the Partner Notification Register) of new HIV-positive results or clients who were receiving enhanced adherence counselling for unsuppressed viral load results were reviewed for activities about Partner notification services. Trained expert elicitors, counselled clients on Partner Notification Services (PNS), and obtained informed consent before proceeding with the service. Those who verbally accepted were interviewed as index cases using structured interview forms. Expert elicitors represent the trained health advisor or provider.

The expert elicitors explained to the index client that all the information collected through the PNS process would be kept confidential and that staff would not reveal index cases' identities when contacting their partners otherwise previously agreed upon. The elicitors first asked a series of questions regarding their demographic characteristics, sexual behaviour, and HIV testing history. Next, they asked index clients how many partners they had in the prior 3 years. If the index case reported no sex partners in the last 3 years, then the health advisors asked them about their most recent sex partner. Following this, the expert elicitors then attempted to collect information about each of the case's acknowledged sex partners, including the partner's demographic characteristics, contact information, the case's relationship to 
the partner, the case's sexual behaviour with the partner, the case's knowledge of the partner's HIV status, and whether the partner had already been notified of the case's HIV result. They also asked about the possibility of IPV from each case of partners identified.

All data were collected using structured interview forms. Records were updated in the monitoring tools and registers. The forms were filled with patient folders in the records department. Index clients were assigned a unique number and their partners with corresponding IDs that had additional sequential numbers allowing partners to be linked to index cases. The expert elicitors went further to enquire how they wanted to notify each partner, either through passive/self-referral approach (the client would like to inform their partners on their own) or through the contract approach (there is an agreed time for the client to inform their partners but not done within the agreed time window the provider can go ahead to inform their partners), or through the dual approach (the expert elicitor and the index client jointly disclose to the partners) or the provider approach (the clients leaves the disclose to the expert elicitor and may also choose not to be named), or through couple counselling (where both clients can be tested together as though the index is not aware of their HIV status), or any other methods (as agreed upon). Each of the methods was explained before they asked the index client to choose a partner notification plan.

If a client chooses a passive or self-referral approach, the expert elicitor goes further to develop the disclosure plan with the index client, including the details of how and where the index client will inform the partner of their HIV status. The language to be used to start the conversation is identified, and the plan will include anticipating the questions the partner will ask, including the response. The index client is encouraged to give the partner referral slip to the partner at the discussion and reminding them that treatment and prevention options are available based on their results. This is rehearsed with the client.

The contract approach follows the same as the passive or self-referral, but a date is agreed by which the index is expected to have notified their partner and refer for HIV testing. The date is not more than 30 days from the date of testing positive. The client is reminded that if their partners do not come for an HIV test by the date, the expert elicitors will call to get his or her permission to contact them directly. The client may also contact the elicitors before then if they are successful or change their mind, or want help.

The expert elicitors and the index client develop a plan to notify the partners together in the dual referral approach. The plan includes where (facility or home) and when to notify them. The provider approach was made by the expert elicitors by calling the partners via telephone using the script for partner notification. The information of the index partner is not shared in the process unless the index client has previously authorized that. If, after three phone call attempts, the partner is not reached, a home visit is made if the partner is within the catchment area. The couple testing approach refers to reoffering couple counselling to the index and partners as though they are jointly tested for the first time. At the same time, other methods refer to any method not previously captured earlier.

Partners who came to the health facility for testing as a result of any of the notification methods were linked to the index case's unique ID number and recorded as notified. Partners who tested positive were commenced on ART and also offered partner notification services as they have now become index clients. All children of index clients less than 15 years were also tested as part of genealogy testing.

The testing data (de-identified) for other testing points or service delivery areas (SDP's)were collected and collated from the HIV Testing Register for the same review period 
from January 2019 to December 2019. The yield (Proportion of New positive gotten from a hundred HTS) from the various service delivery areas was compared with the yield from Index testing.

The data from the structured interview were tabulated on to Excel Office application 2019 MSO (16.0.13530.20418) 3-bit for analysis and interpretation. And generated descriptive statistics for the index clients (Stratification by sex); offer, acceptance, elicitation, and reached rates (the percentage of partners who were successfully notified); the percentage of partners elicited that were HIV counselled and tested, received HIV results, and enrolled into HIV care; the yield from Index testing was compared to the yield from other testing streams in the facility.

\section{Results}

Expert elicitors interviewed 451 index cases within the review period. The study population had a mean age of $43.0(\mathrm{M}=44.1$ years; $\mathrm{F}=42.9$ years), with a range of 15-65 years "Table. 1". The participants were predominantly female $(60.5 \%)$. Newly identified positives accounted for $43 \%$ of participants (194 Clients) and while virally unsuppressed clients accounted for $57 \%$ (257 Clients) "Table. 2". The clients within the review period offered index testing were 450 out of the 451 Index cases (representing 99.1\%; a client who was tested newly positive for HIV died before he was offered PNS). 84.8\% of the clients (382 of 450) accepted Partner Notification Service (PNS).

Participants reported a mean of 1 sexual partner (range, 0-4) in the last 3 years, with the elicitation of 1 Index to 1.2 Partners in the last 3 years (382 Index clients elicited 457 partners). The proportion of partners reached represents 84.9\% (388 of 457 Partners) of the Elicited partners "Table. 3". Most of the Index clients choose Provider referral over the other methods of notification "Figure. 1"; Provider referral (46.4\%), Client referral (37.9\%), Contract referral (8.2\%), and then Dual referral (7.5\%). Of the 388 Clients that were notified, 97 (25\%) were previously known HIV positive clients on Anti-retroviral drugs, 226 (58.2\%) had HIV test done and received results, while 65 (16.6\%) did not present to the clinic for HIV testing despite been notified of their exposure "Figure. 3".

Table 1. Categorized Age and Sex distribution of Client Offered PNS

\begin{tabular}{|l|l|l|l|}
\hline Age & Male & Female & Total \\
\hline $15-19$ & 1 & 4 & 5 \\
\hline $20-24$ & 0 & 15 & 15 \\
\hline $25-29$ & 10 & 47 & 57 \\
\hline $30-34$ & 20 & 69 & 89 \\
\hline $35-39$ & 34 & 62 & 96 \\
\hline $40-44$ & 31 & 44 & 75 \\
\hline $45-49$ & 33 & 19 & 52 \\
\hline $50+y r s$ & 49 & 12 & 61 \\
\hline Grand Total & $\mathbf{1 7 8}$ & $\mathbf{2 7 2}$ & $\mathbf{4 5 0}$ \\
\hline
\end{tabular}

Table 2. Proportions of Index Clients

\begin{tabular}{|l|l|}
\hline New Positive Index & 194 \\
\hline Unsuppressed Index & 257 \\
\hline Total Index & 451 \\
\hline
\end{tabular}


Table 3. PNS Summary Table

\begin{tabular}{|l|l|l|l|}
\hline Indicator & Male & Female & Total \\
\hline \# of Index Clients & 178 & 273 & 451 \\
\hline \# Offered PNS & 178 & 272 & 450 \\
\hline \# Accepted PNS & 150 & 232 & 382 \\
\hline \# Elicited & 240 & 217 & 457 \\
\hline \# Reached & 199 & 189 & 388 \\
\hline \# Notified & 156 & 167 & 323 \\
\hline \# Previously Know Positives & 46 & 51 & 97 \\
\hline \# Tested & 110 & 116 & 226 \\
\hline \# Newly identified Positive & 24 & 38 & 62 \\
\hline \# New Negatives & 86 & 78 & 164 \\
\hline
\end{tabular}

The yield from Index testing alone was 27\% (representing 62 newly identified HIV positive results gotten from 226 tests done) "Figure. 4". All newly identified positive clients were commenced on Anti-retroviral drugs. While within the same review period, yield from other streams is as follows: $10.1 \%$ for Voluntary Counselling and Testing, 2.9\% in TB clinic, $1 \%$ at under 5 Paediatric Clinic, $0.8 \%$ for Provider Initiated Counselling and Testing, $0.7 \%$ at Antenatal Clinic (ANC 1), $0.6 \%$ at Emergency Clinic, and $0.2 \%$ following Post ANC 1(postbooking, during labour, or post-partum).

A total of 62 children below the age of $15 y e a r s$ were Elicited from 382 Index clients, 93.5\% (58 Children) were reached for HIV testing services, but $81 \%$ (47 Children) presented for testing: 1(2.1\%) Child was a previously known HIV positive client already on ART, 4 (8.5\%) children tested newly positive to HIV and were commenced on ART, and 42 (89.4\%) Children tested non-reactive to HIV.

Total HIV positivity rate (Old + New Positives) among elicited clients who presented at the clinic following the notification of their exposure was 49.3\% (159 of 323 Clients) "Figure. 3". Case-finding efficacy was slightly higher in females than males; Expert elicitors interviewed an average of $7.3(\mathrm{M}=7.4, \mathrm{~F}=7.2)$ Index cases to identify 1 new HIV-positive case.

\section{Discussion}

The finding showed that the facility was able to offer Partner Notification Services (PNS) to all new HIV-positive clients and clients with unsuppressed viral load results. The incorporation of PNS into the structure Enhanced Adherence Counselling (EAC) is noteworthy. It is, however clear that HIV PNS shows potential benefits for increasing HIV case identification as well as increasing awareness of possible exposure to HIV as compared to other studies [17, 18] and thus lead to a significant reduction in the transmission of HIV when all clients are on Ante-retroviral therapy [19].

The study also showed a relatively high level of acceptance of the PNS, with most of the clients opting for Provider referral. However, the elicitation is lower than observed from other studies/countries (which is 1:1.8) [20]. This study also aligns with several others, which supports the rational use of resources in the face of dwindling resources for health [21]. Therefore, to maximally use the HIV test kit, which Nigeria has been experiencing shortages recently, priority should be given to Index testing, which gives a yield up to $27 \%$ only closely followed by $10 \%$ at VCT.

The study limitations include the fact that findings are derived from program evaluation 
and not a randomized controlled trial. Therefore, we can't compare with any variation of the number of clients notified, tested and/or linked to care in the absence of the program. Although the study screened for Intimate Partner Violence from the clients elicited, it did not follow up to record how many incidents of violence occurred as a result of the disclosure, whether as partnership dissolution or the loss of emotional or financial support.
Despite these limitations, the results show that implementing PNS can be highly effective in identifying persons with HIV infection and linking them to care. The strategy is costeffective, sustainable, and may be easily incorporated into the daily running of the healthcare facility in many developing nations like Nigeria [22].

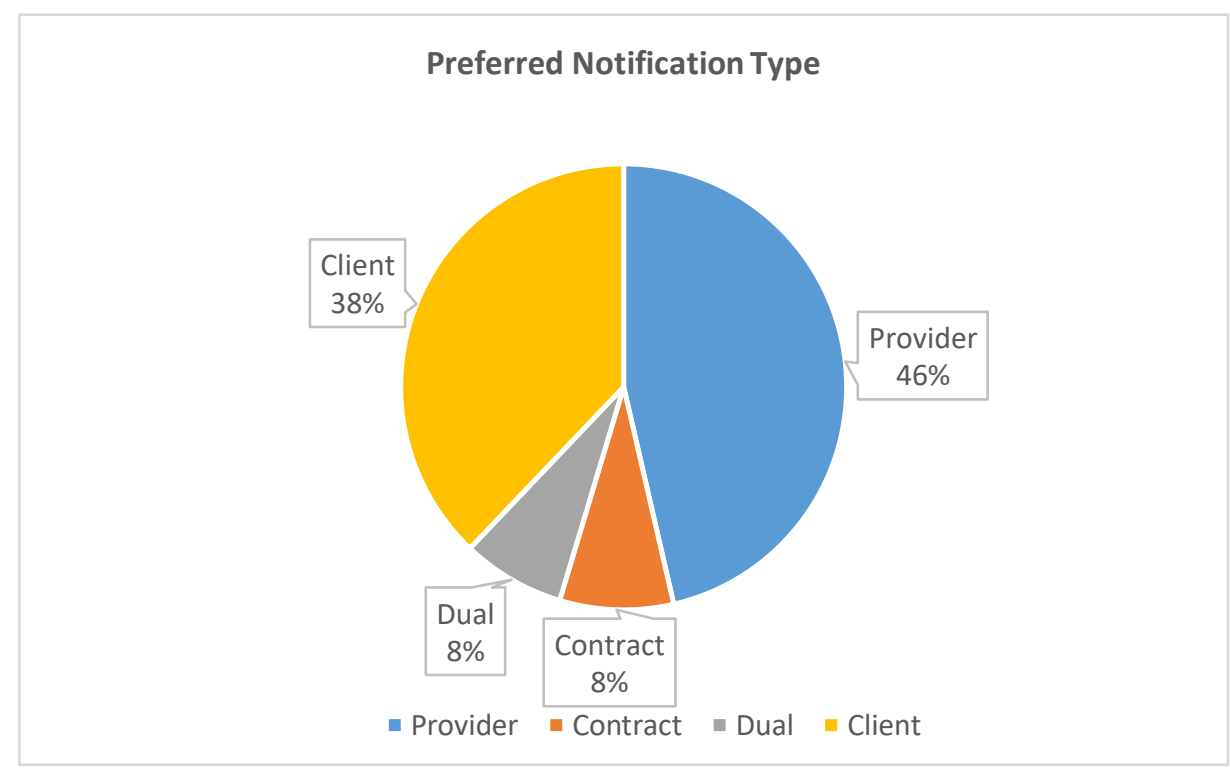

Figure 1. Preferred Notification Type

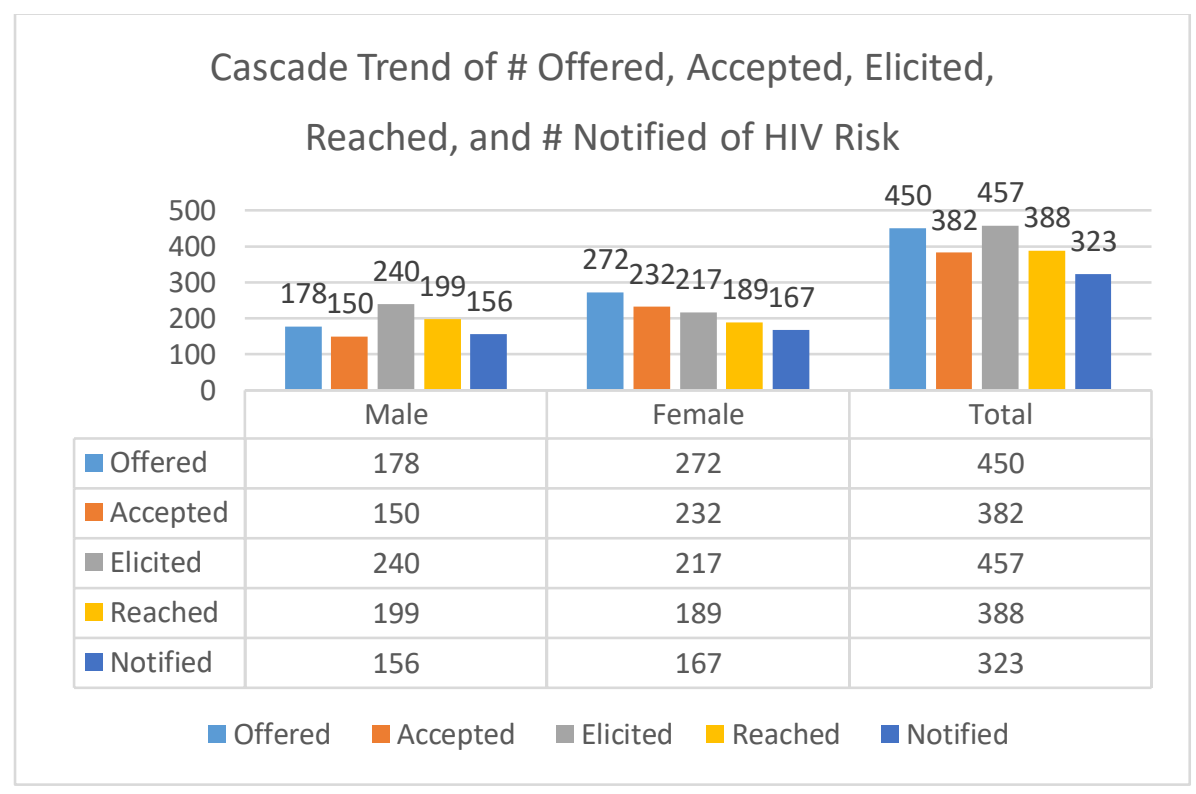

Figure 2. Cascade Trend of \# Offered, Accepted, Elicited, Reached, and \# Notified of HIV Risk 


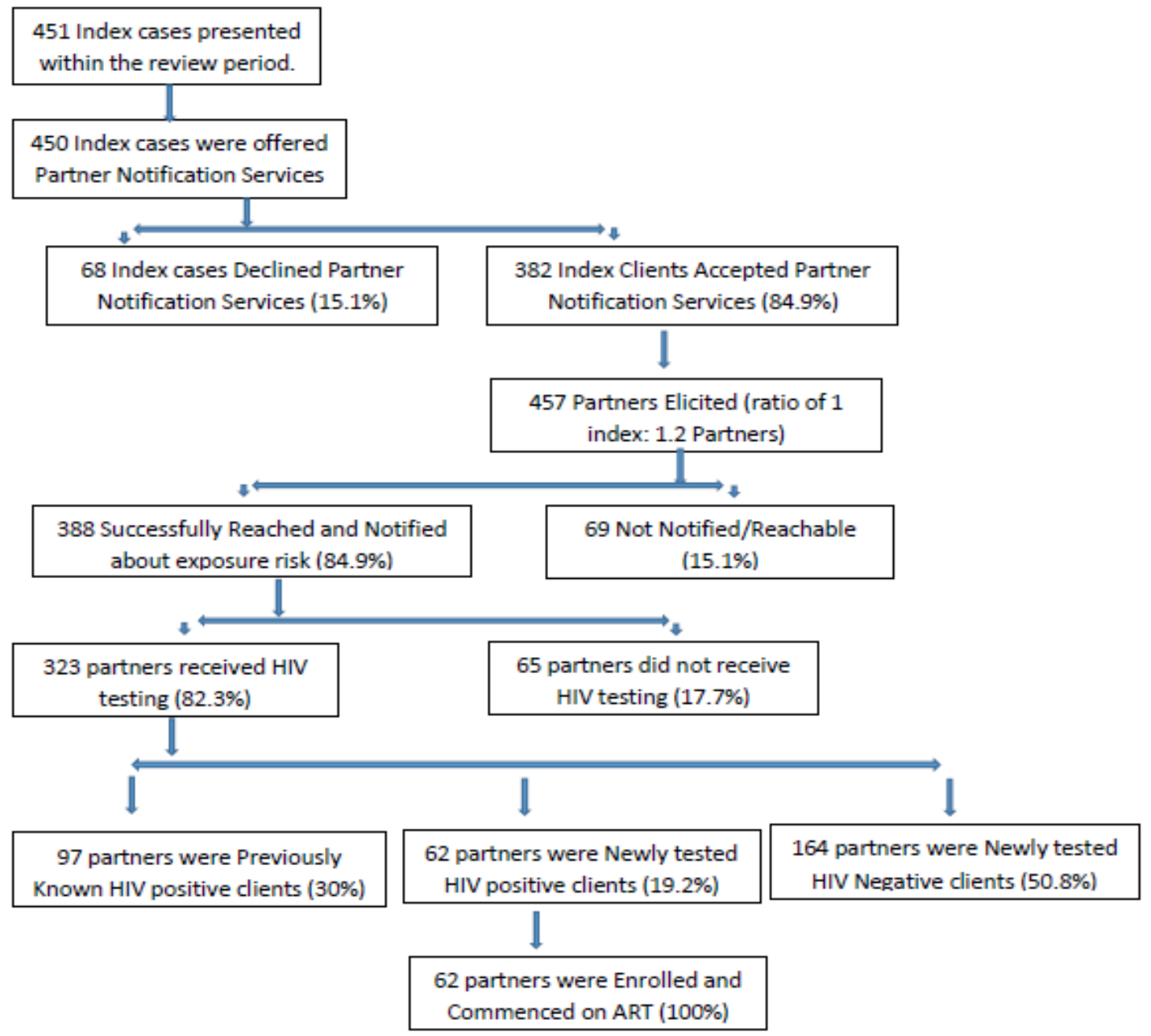

Figure 3. PNS Flow Chart

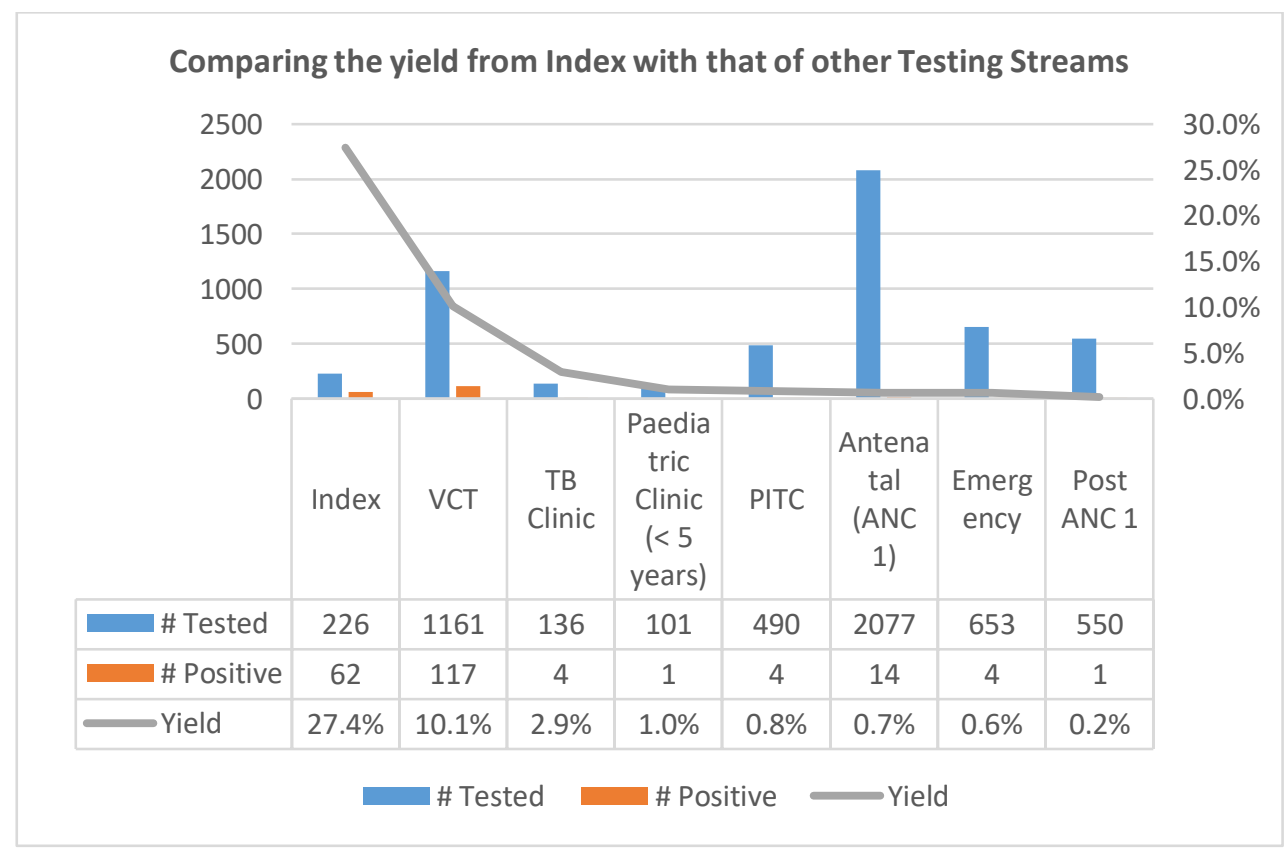

Figure 4. Comparing the yield from Index with that of other Testing Streams 


\section{Conclusion}

Early identification and timely linkage to care are essential to improve the outcomes of persons living with HIV. In our study, almost half of clients elicited during the implementation of Partner Notification Services were infected with HIV (either as new or previously known clients). Partner Notification Services/Approach for Index testing is acceptable, feasible and facilitates the identification and timely linkage to care of HIV-infected persons to HIV treatment services. The adoption of PNS in routine HTS will accelerate the achievement of the first 90 of the UNAIDS goal, which is the entry point to care and treatment for HIV-positive people. PNS is implementable in all levels of health facilities

\section{References}

[1] Bashorun, A., Nguku, P., Kawu, I., Ngige, E., Ogundiran, A., Sabitu, K., Nasidi, A., \& Nsubuga, P. (2014). A description of HIV prevalence trends in Nigeria from 2001 to 2010: what is the progress, where is the problem? The Pan African Medical Journal, $\quad 18$ (Suppl 1 ), 3. https://doi.org/10.11694/pamj.supp.2014.18.1.4608. [2] Aids, N., Programme, C., \& Ministry of Health, F. (2016). National Guidelines for HIV Prevention Treatment and) Care 2016). https://aidsfree.usaid.gov/sites/default/files/2016_nig eria_natl_guidelines_hiv_treat_prev.pdf.

[3] Nigeria HIV/AIDS Indicator and Impact Survey Partners. (n.d.). Retrieved May 23, 2021, from www.health.gov.ng.

[4] Federal Ministry of Health and UNAIDS. (2019).

New survey results indicate that Nigeria has an HIV prevalence of $1.4 \%$. 2030 Ending the AIDS Epidemic, March, 1-3. https://reliefweb.int/sites/reliefweb.int/files/resource s/20190314_PR_Nigeria_en.pdf.

[5] The Value, Importance, and Oversight of Health Research - Beyond the HIPAA Privacy Rule - NCBI Bookshelf. (n.d.). Retrieved March 31, 2020, from https://www.ncbi.nlm.nih.gov/books/NBK9571/

\section{Competing Interest}

The authors have no conflict of interest in this study.

\section{Acknowledgements}

We would like to appreciate everyone that has contributed to the journey of this success. It has truly been a great experience. More thanks to the entire University of Abuja Teaching Hospital and Institute of Human Virology Nigeria community for giving us that chance to undertake this research work at the University of Abuja Teaching Hospital. Thank you.

[6] WHO | HIV testing services. (2019). WHO. https://www.who.int/hiv/topics/vct/about/en/.

[7] WHO. (2018). WHO Recommends Assistance for People with HIV to Notify Their Partners. https://sahivsoc.org/Files/HIVPN-Policybrief-

Globalversion.pdf.

[8] Partner Notification Services | Engage in Small Talks | PIC | Campaigns | Act Against AIDS | CDC. (n.d.). Retrieved May 25, 2019, from https://www.cdc.gov/actagainstaids/campaigns/pic/t alks/partner-notification.htmll.

[9] Reaching Families through Index Case Testing in Malawi. (n.d.). Retrieved May 25, 2019, from https://medium.com/@MSHHealthImpact/reachingfamilies-through-index-case-testing-in-malawif47f14046af3.

[10]Fontaine, J., Sfetcu, O., Van De Laar, M., Martin-Hilber, A., Röllin, A., Redmond, S., Scott, P., Trelle, S., \& Cassell, J. (n.d.). Public health benefits of partner notification for sexually transmitted infections and HIV. https://doi.org/10.2900/85700.

[11] Index and Partner Notification Testing Toolkit - PEPFAR Solutions Platform (BETA). (n.d.). Retrieved May 25, 2019, from https://www.pepfarsolutions.org/tools-

2/2018/4/11/index-and-partner-notification-testingtoolkit. 
[12] Khongonyowa, L., Schouten, E., \& Aziz, A. (2018). Index Case Testing: A Promising Strategy for Achieving HIV epidemic control Background. https://www.msh.org/sites/default/files/cdc_-

_index_case_brief.pdf.

[13] AIDSFree. (n.d.). Partner Notification: A handbook for designing and implementing programs and services. Retrieved May 25, 2019, from https://aidsfree.usaid.gov/sites/default/files/resources /2018.6.19_pn-handbook_0.pdf.

[14] Com, I. (2015). Consolidated Guidelines On. http://www.who.int/hiv/pub/vct/hiv-self-testing-

guidelines/en/.

[15]Dalal, S., Johnson Cheryl, Fonner Virginia, Kennedy Caitliin E, Siegfried Nandi, Figueroa Carmen, \& Baggaley Racheal. (2017). WHO | Assisted HIV partner notification services: a systematic review and meta-analysis. WHO. http://www.who.int/hiv/pub/journal_articles/partnernotification-systematic-review/en/.

[16]Improving HIV Case-Findging using Partner Notification Services: A Snapshot from Zambia. (n.d.). Retrieved May 25, 2019, from https://www.fhi360.org/sites/default/files/media/doc uments/zpct-improving-hiv-case-finding-May18.pdf. [17] Kariuki, R. M., Rithaa, G. K., Oyugi, E. O., \& Gachathi, D. M. (2020). What is the level of uptake of partner notification services in HIV testing in selected health facilities in Gatanga Sub County, Muranga County - Kenya; A retrospective study. BMC Infectious Diseases, 20(1), 1-7. https://doi.org/10.1186/s12879-020-05146-9.
[18]Index Case Testing: A Promising Strategy for Achieving HIV Epidemic Control | Management Sciences for Health. (n.d.). Retrieved May 25, 2019, from https://www.msh.org/resources/index-casetesting-a-promising-strategy-for-achieving-hivepidemic-control.

[19] Albert, J., Berglund, T., Gisslén, M., Gröön, P., Sönnerborg, A., Tegnell, A., Alexandersson, A., Berggren, I., Blaxhult, A., Brytting, M., Carlander, C., Carlson, J., Flamholc, L., Follin, P., Haggar, A., Hansdotter, F., Josephson, F., Karlström, O., Liljeros, F., Widgren, K. (2014). Risk of HIV transmission from patients on antiretroviral therapy: A position statement Public Health Agency of Sweden and the Swedish Reference Group for Antiviral Therapy. In Scandinavian Journal of Infectious Diseases (Vol. 46, Issue 10, pp. 673-677). Informa Healthcare. https://doi.org/10.3109/00365548.2014.926565.

[20]Linah K. Mwango RN. (2019). Reaching the Unreachable: Early Results from Index Testing in Zambia in the CIRKUITS Project. 10th IAS Conference on HIV Science. http://programme.ias2019.org/PAGMaterial/PPT/14 39_3652/22.07.2019, IAS Conference Reaching the unreached_Lina Mwango.pptx.

[21] Collins, J., \& Rau, B. (n.d.). AIDS in the Context of Development UNRISD Programme on Social Policy and Development, Paper Number 4, December 2000.

[22]HIV Partner Notification Services. (2016). https://www.ncbi.nlm.nih.gov/books/NBK401676/. 